This is the published version of:

Alexandre Jeanneau, Éric Prieto, Nicolas Flagey, Alexis Hill, Kjetil Dohlen, Roser Pello, Laurence Tresse, Sylvie Brau-Nogué, Christophe Yèche, PierreHenri Carton, "Maunakea Spectroscopic Explorer Low Moderate Resolution Spectrograph: paths toward the Preliminary Design Phase," Proc. SPIE 11447, Ground-based and Airborne Instrumentation for Astronomy VIII, 1144795 (13 December 2020)

The published version of the manuscript is also available at https://doi.org/10.1117/12.2576314

Copyright 2020 Society of Photo-Optical Instrumentation Engineers. One print or electronic copy may be made for personal use only. Systematic reproduction and distribution, duplication of any material in this paper for a fee or for commercial purposes, or modification of the content of the paper are prohibited. 


\section{Maunakea Spectroscopic Explorer Low Moderate Resolution Spectrograph: paths toward the Preliminary Design Phase}

Jeanneau, Alexandre, Prieto, Éric, Flagey, Nicolas, Hill, Alexis, Dohlen, Kjetil, et al.

Alexandre Jeanneau, Éric Prieto, Nicolas Flagey, Alexis Hill, Kjetil Dohlen, Roser Pello, Laurence Tresse, Sylvie Brau-Nogué, Christophe Yèche, PierreHenri Carton, "Maunakea Spectroscopic Explorer Low Moderate Resolution Spectrograph: paths toward the Preliminary Design Phase," Proc. SPIE 11447, Ground-based and Airborne Instrumentation for Astronomy VIII, 1144795 (13 December 2020); doi: 10.1117/12.2576314 


\title{
Maunakea Spectroscopic Explorer Low Moderate Resolution Spectrograph: paths toward the Preliminary Design Phase
}

\author{
Alexandre Jeanneau $^{\mathrm{a}}$, Éric Prieto ${ }^{\mathrm{b}}$, Nicolas Flageyc ${ }^{\mathrm{c}}$, Alexis Hill ${ }^{\mathrm{c}}$, Kjetil Dohlen ${ }^{\mathrm{b}}$, Roser Pello ${ }^{\mathrm{b}}$, \\ Laurence Tresse $^{\mathrm{a}}$, Sylvie Brau-Noguéd ${ }^{\mathrm{d}}$, Christophe Yèche ${ }^{\mathrm{e}}$, and Pierre-Henri Carton ${ }^{\mathrm{e}}$ \\ ${ }^{a}$ Univ Lyon, Univ Lyon1, Ens de Lyon, CNRS, Centre de Recherche Astrophysique de Lyon \\ UMR5574, F-69230, Saint-Genis-Laval, France \\ ${ }^{\mathrm{b}}$ Aix Marseille Univ, CNRS, CNES, LAM, Marseille, France \\ ${ }^{\mathrm{c}}$ CFHT Corporation, 65-1238 Mamalahoa Hwy, Kamuela, Hawaii 96743, USA \\ ${ }^{\mathrm{d}}$ CNRS, IRAP, 14 avenue Edouard Belin, 31400 Toulouse, France \\ eIRFU, CEA, Université Paris-Saclay, F-91191 Gif-sur-Yvette, France
}

\begin{abstract}
The Maunakea Spectroscopic Explorer (MSE) is a 10m-class, wide-field (1.5 sq. degree) and high-multiplex (> 3000 fibers) spectroscopic facility that will replace the $3.6 \mathrm{~m}$ Canada-France-Hawaii Telescope. With backend spectrographs operating at low $(R \sim 3000)$, moderate $(R \sim 6000)$ and high $(R \sim 20 / 40 \mathrm{k})$ spectral resolution across the $0.36-1.8 \mu \mathrm{m}$ range, MSE will be poised to address a variety of science questions among which the nature of dark matter, the origin of the elements in the periodic table, the mass of the neutrino, whilst enabling a new era of rapid-response and time-domain astronomy.

This paper is a status report of the MSE Low Moderate Resolution spectrograph design, from the Conceptual Design Review (CoDR) towards the Preliminary Design Phase (PDP).
\end{abstract}

Keywords: Astrophysics - Instrumentation and Methods for Astrophysics

\section{INTRODUCTION}

The Maunakea Spectroscopic Explorer (MSE) is a 10m-class, wide-field (1.5 sq. degree) and high-multiplex (> 3000 fibers) spectroscopic facility that will replace the $3.6 \mathrm{~m}$ Canada-France-Hawaii Telescope. MSE comprises two spectrographs: a High Resolution (HR) spectrograph described in a parent publication*, and a Low and Moderate Resolution (LMR) spectrograph.

With its two configurations (low and moderate resolution), the LMR will have a strong impact on a number of science cases spanning all scales from stellar to cosmological. The LMR has undergone its Conceptual Design Review (CoDR) in 2017 at CRAL, followed with recommendations made by the review panel. In 2019, the high level requirements have been revised on the basis of the answers from a questionnaire sent to the Science Team Working Groups. Trade-off analyses have then been launched.

In this paper we present the design rationale derived from the CoDR report, including the conceptual design and the revised high-level requirements in Sect. 2. The proposed near-IR spectrograph design is discussed in details in Sect. 3. Sect. 4 briefly presents an alternative design based on a cross-dispersion alternative.

\section{DESIGN RATIONALE}

A design rationale has been derived from the CoDR report as well as the revised high level requirements. This rationale is mainly driven by risk-mitigation, in the context of increasingly challenging requirements.

\footnotetext{
Send correspondence to alexandre.jeanneau@univ-lyon1.fr

* Mauna Kea Spectrographic Explorer (MSE): New preliminary design for the multi-object high resolution spectrograph, to be presented during this digital forum.
} 


\section{The Conceptual Design}

The Conceptual Design (Fig. 1) was derived from the AAT/Hector [1,2] layout and optimized in term of cost per spectrum. It is extensively described in [3] and its requirements are summarized in Table 1. The conceptual design meets spectral coverage and resolution requirements (Fig. 2) except for a non-compliance in the blue $(370-460 \mathrm{~nm})$ moderate resolution mode, and complies with most other requirements.

The design consists of a laterally-curved fiber slit $(104 \mathrm{~mm})$, a rectangular field lens gelled to the fibers, an off-axis Schmidt collimator $^{\dagger}(F / 2.08)$, three dichroics and four spectral arms. Three spectral arms cover the optical range, providing both low $(R \sim 3200)$ and moderate $(R \sim 5400)$ spectral resolution. In order to switch resolution, the off-axis Schmidt corrector lens and the grating (low resolution) are exchanged for a large sapphire grism (moderate resolution), with a corrector bonded to the front face. The remaining spectral arm provides NIR coverage over the YJ bands at low resolution $(R \sim 3500)$, or over the $\mathrm{H}$ band at moderate resolution $(R \sim 5900)$. This $\mathrm{H}$ band capability requires a cryogenic optical train $(200<T<220 \mathrm{~K})$ from the slit to the detector, so as to minimize the thermal background.

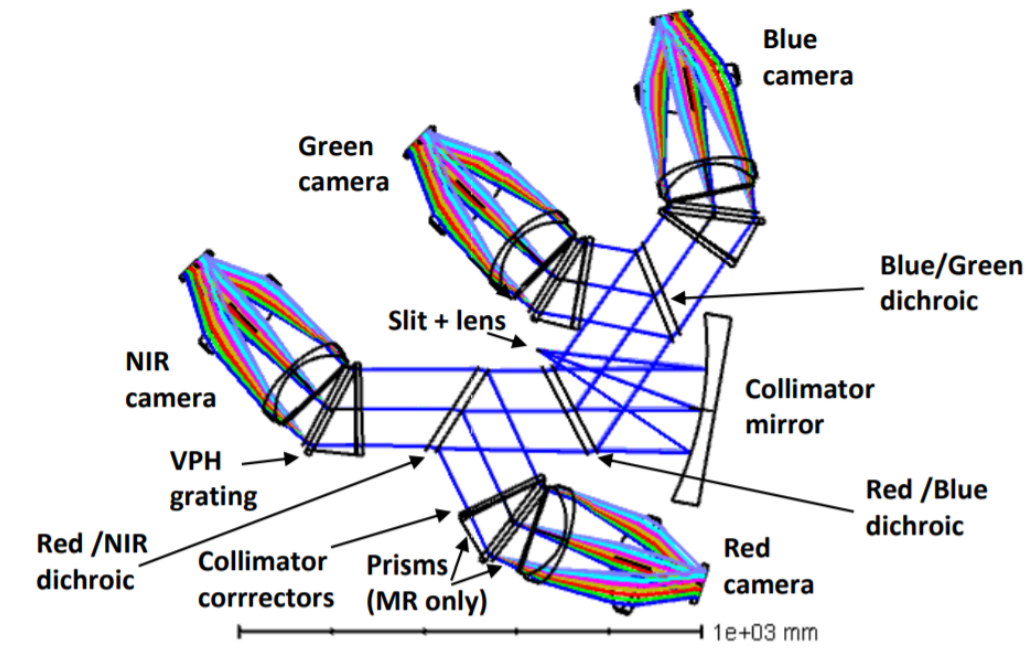

Figure 1. Optical layout of the CoDR LMR spectrograph [3].

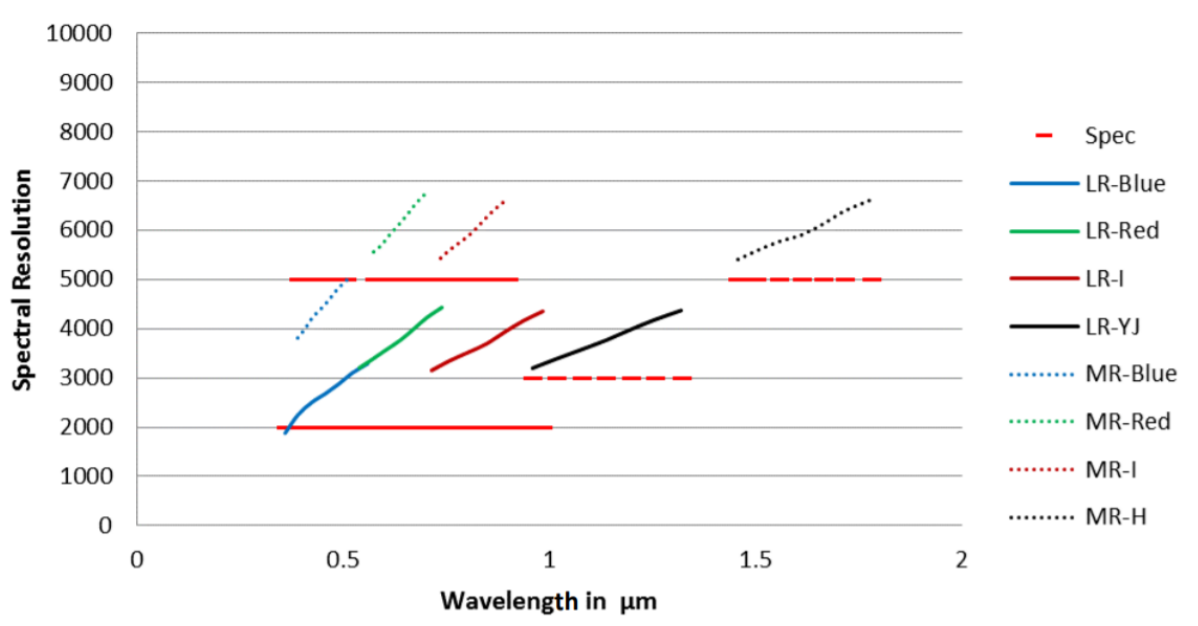

Figure 2. CoDR LMR spectral resolution [3], excluding manufacturing and alignement errors.

The four transmissive cameras are very fast $(F / 1.2)$, so as to fit the required number of spectral resolution

\footnotetext{
${ }^{\dagger}$ The selected collimator speed of $F / 2.08$ corresponds to a diameter of $10.42 \mathrm{~m}$ at the primary mirror.
} 
elements on the privileged $4 \mathrm{k} \times 4 \mathrm{k}, 15 \mu \mathrm{m}$-detector format ${ }^{\ddagger}$. Considering both detector format and overall de-magnification, six replicated spectrograph units are needed to achieve the required multiplexing $(\sim 3200)$. The camera design has only two doublets and a field lens per spectral arm, yet with highly aspheric shapes. With aspheric departures up to $2.4 \mathrm{~mm}$ over a $175 \mathrm{~mm}$ collimated beam diameter, manufacturing and above all aligning such lenses would prove difficult. Besides, the overall optical layout is tightly packaged, raising serious concerns about the assembly phase. In light of these risks, the review panel recommended to pursue alternate optical designs.

\section{Revised High-Level Requirements}

Following CoDR, the high-level requirements have been revised in 2019, on the basis of a questionnaire answered by $\sim 60$ members of the Science Team. The revised requirements are related to four metrics: spectral coverage, spectral resolution, sensitivity and multiplexing. Until now, only spectral coverage and spectral resolution have flowed down into proper design requirements: sensitivity (i.e. throughput) is optimized on a best effort basis, and multiplexing relates to the number of replicated spectrograph units with a marginal impact on their design. The high-level requirements (both conceptual and revised) are presented in Table 1.

Table 1. High-level requirements (both conceptual and revised) for the Low and Moderate Resolution spectrograph. The main revisions are highlighted in bold characters. Some high-level design parameters are added underneath the high-level requirements.

\begin{tabular}{|c|c|c|c|c|}
\hline \multirow{2}{*}{ Requirement } & \multicolumn{2}{|c|}{ Conceptual (2017) } & \multicolumn{2}{|c|}{ Revised (2019) } \\
\hline & VIS & NIR & VIS & NIR \\
\hline Spectral coverage & \multicolumn{2}{|c|}{$\begin{array}{c}0.36-1.3 \mu \mathrm{m} \\
\text { OR } \\
0.36-0.95 \mu \mathrm{m} \text { and } 1.5-1.8 \mu \mathrm{m}\end{array}$} & $0.36-1.0 \mu \mathrm{m}$ & $\begin{array}{c}1.0-1.3 \mu \mathrm{m} \\
\text { AND } \\
1.45-1.8 \mu \mathrm{m}\end{array}$ \\
\hline $\begin{array}{l}\text { Spectral resolution } \\
\text { (low) }\end{array}$ & $2500<R_{\text {avg }}<3000$ & $R_{\min }>3000$ & $3000<R_{\text {avg }}<3500$ & $R_{\min }>3000$ \\
\hline $\begin{array}{l}\text { Spectral resolution } \\
\text { (moderate) }\end{array}$ & \multicolumn{2}{|c|}{ 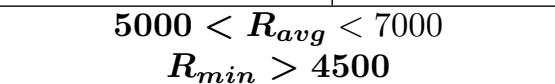 } & $\begin{array}{c}\mathbf{5 0 0 0}<\boldsymbol{R}_{\text {avg }}<6000 \\
\boldsymbol{R}_{\text {min }}>\mathbf{5 0 0 0}\end{array}$ & $\begin{array}{c}\mathbf{6 0 0 0}<\boldsymbol{R}_{\text {avg }}<7000 \\
\boldsymbol{R}_{\text {min }}>\mathbf{5 5 0 0}\end{array}$ \\
\hline Multiplexing & \multicolumn{2}{|l|}{3249} & 2166 (TBD) & 1083 \\
\hline Sensitivity & \multicolumn{2}{|c|}{$\operatorname{mag}_{A B}>24$} & \multicolumn{2}{|c|}{$\operatorname{mag}_{A B}>24(>23$ in $\mathrm{H}$ band $)$} \\
\hline Cross-talk & \multicolumn{4}{|c|}{$2 \%$ (goal: $1 \%$ ) } \\
\hline On-sky fiber diameter & \multicolumn{4}{|c|}{$1.0 \operatorname{arcsec}$} \\
\hline Fiber output F-number & \multicolumn{4}{|c|}{$F / 2.08$} \\
\hline
\end{tabular}

The revised high-level requirements are increasingly stringent in terms of simultaneous coverage and resolution, especially in the NIR moderate resolution mode. The conceptual design was able to accommodate both visible and one NIR band (YJ at low resolution or $\mathrm{H}$ at moderate resolution), yet with a non-compliant moderate resolution according to the revised requirements. This prompts to add spectral arms and make all cameras significantly faster $(\sim F / 1$ or less).

However, fitting more spectral arms on a unique layout is not an option, as the opto-mechanical packaging of the conceptual design was already too tight according to the review panel. Splitting the NIR from the visible is interesting from a technical standpoint, as doing so minimizes the cooled mass while allowing to increase the overall number of spectral arms. Waiving the constraint to fit the full bandpass on a single layout also allows to introduce larger detectors or curved detectors in the visible paths. Besides, the questionnaire revealed that the scientific need for simultaneous visible and NIR coverage is relatively low.

Therefore, we explored a trade-off consisting in feeding a NIR spectrograph with dedicated fibers, representing a third of the LMR fibers (1083). Depending on the focal plane design, additional fiber positionners may be added so as to restore a 3200 multiplex capability in the visible.

\footnotetext{
${ }^{\ddagger}$ This detector format is a constraint, especially in the NIR.
} 
In the remainder of the paper, we propose a design for the moderate-resolution NIR spectrograph, which remains the most critical part of the LMR with both harsh requirements and reduced design options. Another on-going trade-off analysis focuses on the possibility to achieve a low resolution mode numerically, by binning moderate resolution spectra. This second trade-off analysis (outside the scope of this paper) may temper the additional cost/complexity resulting from additional spectral arms equipped with grating exchange mechanisms.

\section{PROPOSED NEAR-INFRARED SPECTROGRAPH DESIGN}

\subsection{Main parameters}

The proposed moderate-resolution design is largely inspired by the so-called WonderCamera, developed for the ESO-VLT/MOONS instrument $[4,5]$. We summarize here the main scaling laws driving the spectrograph design:

Fiber core diameter (on detector)

Fiber pitch (on detector)

Slit length

Simultaneous wavelength coverage

$$
\begin{aligned}
& d_{f i b}=3.4 \mathrm{px}\left(\frac{\theta_{f i b}}{1 \operatorname{arcsec}}\right)\left(\frac{F_{c a m}}{0.95}\right)\left(\frac{\eta_{F R D}}{0.95}\right)^{-1} \\
& \Delta_{f i b}=5.5 \mathrm{px}\left(\frac{\theta_{f i b}}{1 \operatorname{arcsec}}\right)\left(\frac{F_{c a m}}{0.95}\right)\left(\frac{\eta_{F R D}}{0.95}\right)^{-1} \\
& L_{\text {slit }}=98 \mathrm{~mm}\left(\frac{N_{f i b}}{541}\right)\left(\frac{\theta_{f i b}}{1 \operatorname{arcsec}}\right)\left(\frac{\eta_{F R D}}{0.95}\right)^{-1}\left(\frac{F_{c o l l}}{2.08}\right) \\
& \lambda_{\text {max }}-\lambda_{\min }=0.26 \lambda_{\min }\left(\frac{N_{p x}}{4096}\right)\left(\frac{R_{\min }}{5500}\right)^{-1}\left(\frac{d_{f i b}}{3.4 \mathrm{px}}\right)^{-1}
\end{aligned}
$$

where $\theta_{f i b}$ is the on-sky fiber diameter, $F_{\text {coll }}$ and $F_{c a m}$ respectively the collimator and camera focal ratio, $\eta_{F R D}$ the focal ratio degradation factor due to the fibers, $N_{f i b}$ the number of fibers per spectrograph unit, $N_{p x}$ the number of pixels along the spectral axis and $R_{\min }$ the spectral resolution at the blue edge of the spectral arm.

\subsection{Catadioptric design}

The design rationale derived from the CoDR report and the revised high-level requirements lead to keep a similar collimator design and introduce fast $(F / 0.95)$ catadioptric cameras in two NIR units. Considering the current fiber allocation for NIR, each unit receives light from 541 fibers, although the detector chips could accommodate more. The resulting design comprises a curved ${ }^{\S}$ fiber slit $(98 \mathrm{~mm})$, a rectangular field lens gelled to the fibers, an on-axis Schmidt collimator $(F / 2.08)$, a dichroic and two spectral arms to accommodate respectively the YJ and $\mathrm{H}$ bands. The optical layout and the spot diagram are shown in Figs. 3 and 4.

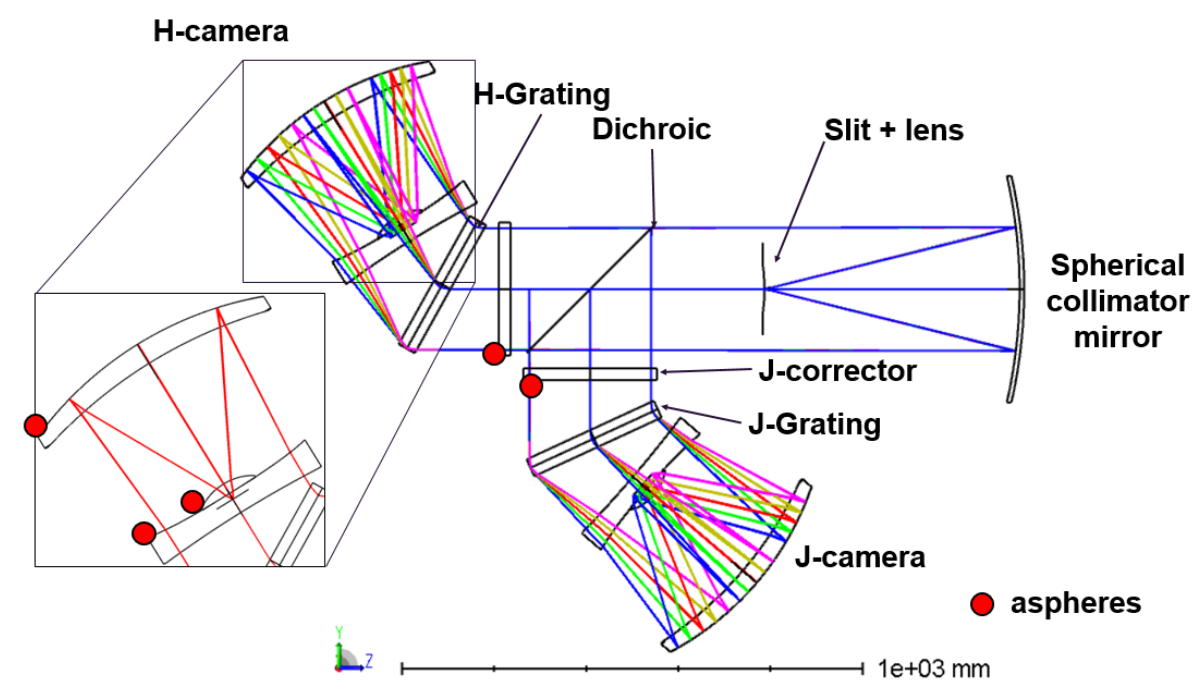

Figure 3. Optical layout of the proposed NIR design for the LMR.

\footnotetext{
${ }^{\S}$ We intend to introduce a lateral sag as in the CoDR design, in order to compensate spectral curvature on the detector.
} 

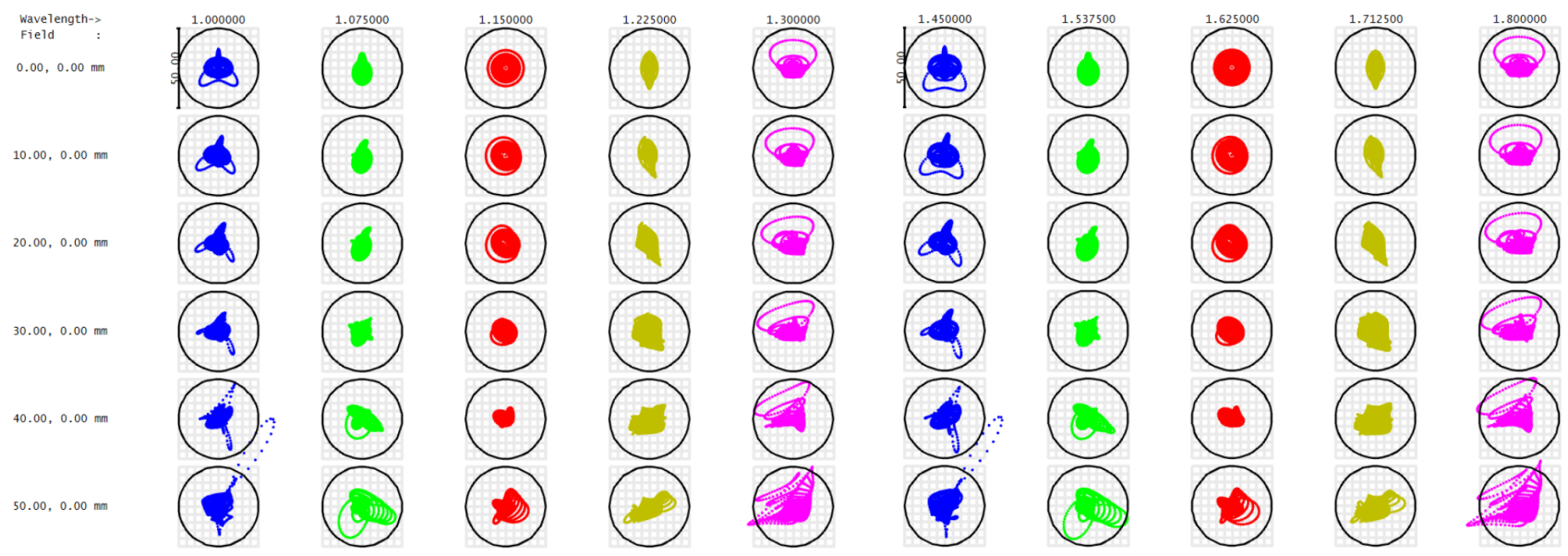

Figure 4. Spot diagram matrix of the proposed catadioptric NIR design for the LMR. The different wavelengths and fiber positions span the horizontal and vertical axes, respectively. The fiber diameter is represented as a black circle, for comparison.

The camera design comprises only three elements made of fused silica: a first lens, a Mangin mirror and a second lens. The first lens is centrally obscured by the detector and the second lens, leading to considerable obstruction losses $(\sim 16 \%)$ despite the large beam diameter $(265 \mathrm{~mm})$. However, these losses are partially compensated by the exceptionally small number of optical elements. The Mangin mirror allows for a slight image quality improvement with respect to a traditional mirror, yet with a potential increase in stray light which prompts for a trade-off.

A first sensitivity analysis showed that the most stringent positioning and stability tolerances are reasonable: $\sim 50 \mu \mathrm{m}$ in translation and $\sim 1$ arcmin in tilt for the most sensitive elements. Finally and as shown in [4], the camera can be made temperature-invariant with a low-CTE structure, since all optical elements are made of fused silica. This feature could allow the alignment process to take place at ambient temperature. As the conceptual design, the proposed design needs to be cooled down between 200 and $220 \mathrm{~K}$ in order to minimize thermal background.

\subsection{Expected end-to-end performance}

In this subsection, we present a first assessment of the end-to-end performance we expect by design. Lacking a thorough tolerancing analysis, we assumed comfortable margins for manufacturing and alignment.

Both spectral resolution and cross-talk are computed by fitting a gaussian to the fraction of encircled energy $(30,50,80,95$ and $99 \%)$. This gaussian function is then convolved by another gaussian accounting for manufacturing and alignement margins, whose FWHM is $50 \%$ narrower. The resulting 'as-built' PSF is then convolved by the geometrical fiber image and re-sampled on $15 \mu \mathrm{m}$ bins, resulting in a simulated fiber image as seen by the detector. The overall process is depicted in Fig. 5.

\subsubsection{Spectral resolution}

In order to assess spectral resolution, defined as $R=\lambda / \Delta \lambda$ where $\Delta \lambda$ is the FWHM of the LSF at wavelength $\lambda$, we collapse the simulated detector image along the spatial direction, by summing rows of five pixels. The FWHM of the resulting LSF profile is estimated using a gaussian fit. The expected spectral resolution as a function of wavelength is shown in Fig. 6.

The proposed catadioptric design is compliant over most of the NIR spectral range at moderate resolution, yet with an insufficient minimal spectral resolution in the YJ band $\left(R_{\min } \sim 4800\right.$ instead of 5500$)$. Reaching such minimal resolution while accommodating this spectral range would require a faster camera $(F / 0.85)$, an additional spectral arm or a different detector format $\mathbb{I}$.

\footnotetext{
${ }^{\mathbb{I}}$ One could use a mosaic of $2 \mathrm{k} \times 2 \mathrm{k}, 18 \mu \mathrm{m}$-detectors. However, the gap between detectors would result in a waveband loss and the larger focal plane would increase the overall obstruction losses.
} 

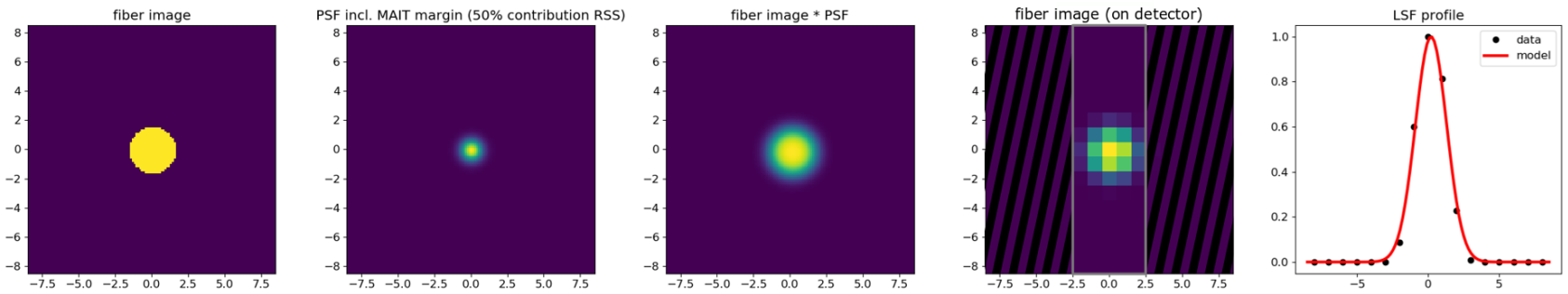

Figure 5. Computation process of a simulated fiber image on the detector. From left to right: a perfect geometrical fiber image, the geometrical PSF fitted and convolved so as to account for manufacturing and alignment errors, the resulting 'as-built' fiber image, the fiber image as sampled by the detector and a LSF profile extracted from the latter. The dashed area of the simulated fiber image is used for cross-talk estimation, whereas the ensquared area is used to assess spectral resolution.

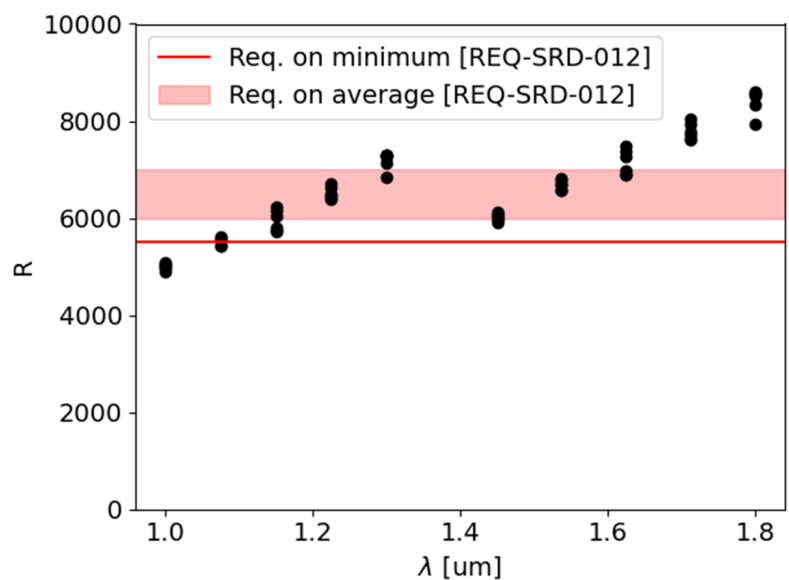

Figure 6. Expected spectral resolution (including margins).

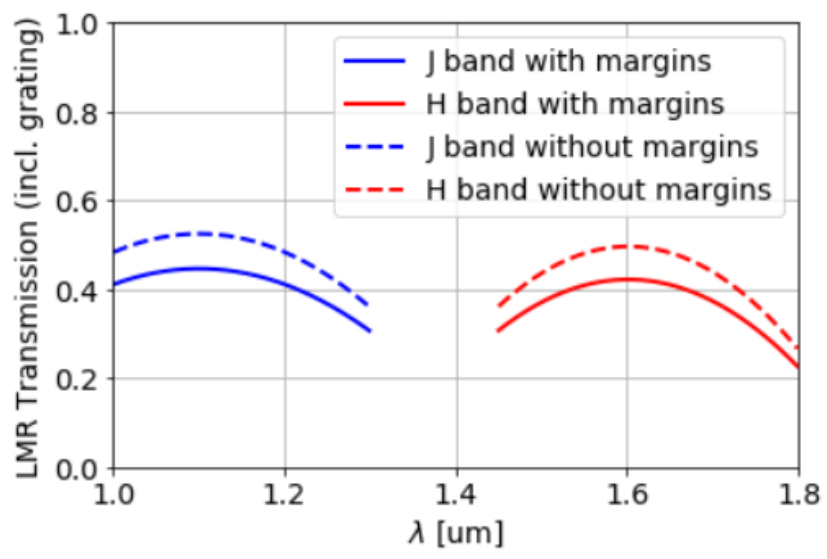

Figure 7. Expected transmission with and without margins (respectively solid and dashed lines).

\subsubsection{Crosstalk}

Fiber-to-fiber crosstalk is estimated as the energy fraction falling outside of the five-pixel-wide band allocated to the simulated fiber spectrum. This preliminary estimate, shown in Fig. 8, does not include straylight nor charge diffusion effects.

The overall crosstalk, including manufacturing and alignment margins, achieves the $<1 \%$ goal over most of the slit, except for the reddest wavelengths of the farthest fibers which still comply with the $<2 \%$ requirement.

\subsubsection{Transmission}

The main contributors to the LMR transmission are inevitably the large obstruction $(\sim 16 \%$ including optomechanical margins to accommodate a cold finger or spiders) inherent to the catadioptric camera design $\|$ and grating efficiency. We use theoretical VPH grating transmission curves from CoDR (peak efficiency $\sim 90 \%$ ) and assume respectively $98.0 \%$ and $99.0 \%$ efficiency for reflective and anti-reflective coatings. We conservatively assume the detector to reach 'spec-sheet' quantum efficiency (80\%), even though as-built detectors can go as high as $\sim 90 \%$. Finally, we consider a $15 \%$ 'contingency' margin.

Under these assumptions, we expect the NIR LMR units to reach an average efficiency of $40 \%$ in the YJ band and $36 \%$ in the $\mathrm{H}$ band, with respectively $44 \%$ and $41 \%$ peak efficiency (Fig. 7). Considering similar assumptions, the CoDR design reaches 53\% (YJ band) and 45\% (H band) peak efficiency.

\footnotetext{
"We did not take into account slit obstruction in this preliminary estimate, which is relatively small with respect to detector obstruction and partially mitigated by it.
} 

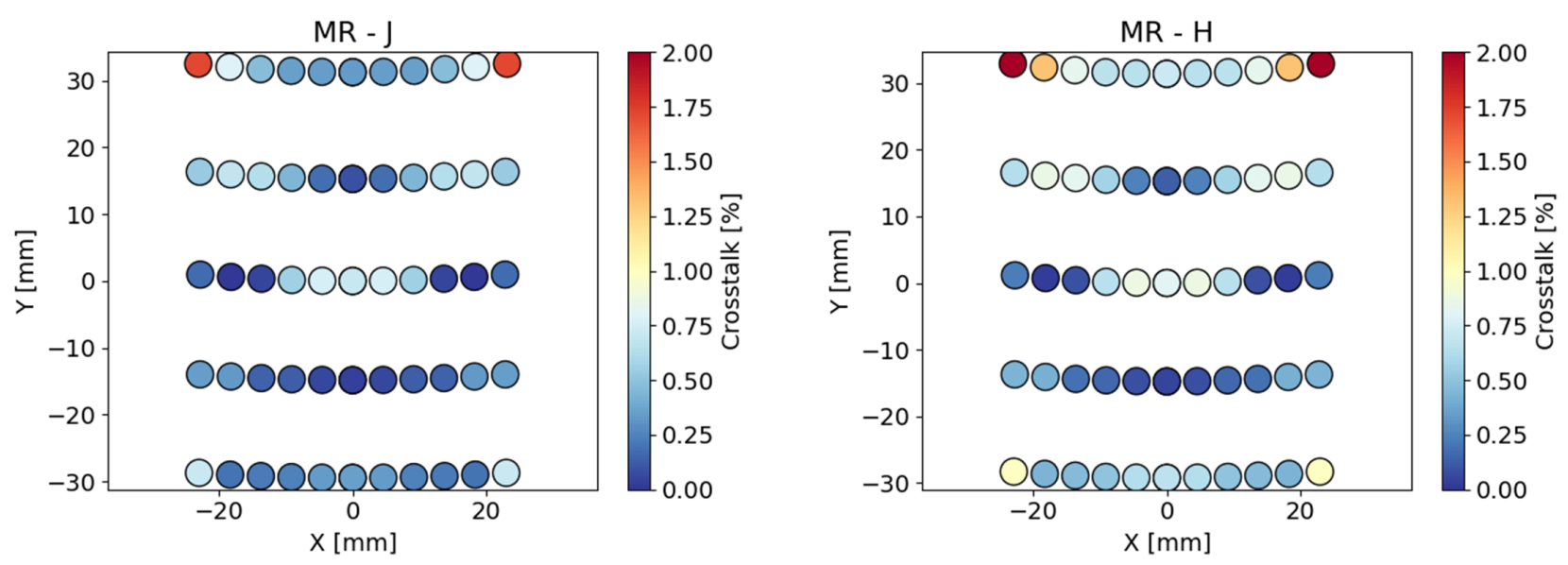

Figure 8. Expected crosstalk between a fiber and its neighbours (including margins). With the current fiber allocation for NIR, each unit receives light from 541 fibers, although the detector chips could accommodate more.

\section{ALTERNATIVE DESIGN BASED ON CROSS-DISPERSION}

One could question the fact to have dichroic elements in the spectrograph. Barden [6] shows a path by using the well known cross dispersion technic. We propose here a concept with an unique camera covering the $\mathrm{J}$ and $\mathrm{H}$ bands on the same $4 \mathrm{kx} 4 \mathrm{k}$ detector. Each instrument could be fed with approximately 400 fibers. The 3 channels could fulfill the LMR needs. The optical layout is shown in Fig. 9.

We implement a catadioptric camera and collimator. Finally, we use the capability of a variable line density grating on the grism to help in the aberration correction.

As mentioned above, the instrument shall be fully cryogenic (from the fiber slit to the detector). In this preliminary concept, we choose to have a straight optical path. The advantage for this option is to have the possibility to select a cylindrical cryostat. This point could help in cost and risk mitigation. The drawback is the necessity to have a large and massive prism.

During later phase, we will balance the different cost and risk at instrument level between optics, mechanics and thermal difficulties. Efforts will be pursued to minimize the overall size which is currently quite large (2.5-m long). Nevertheless, with similar performance to the nominal concept, the optical performance is quite good. The spot diagrams are shown in Fig. 10.

\section{CONCLUSION}

Due to an increasingly challenging spectral resolution over a widening simultaneous bandpass, the LMR spectrograph design requires an increasing number of fast cameras (faster than $F / 1$ ). In this paper, we have explored a trade-off consisting in splitting the visible arms and the NIR arms, and proposed a catadioptric design for the latter. The proposed design reaches the required spectral resolution over the bandpass except for a $13 \%$ discrepancy at $1.0 \mu \mathrm{m}$, while preserving comfortable margins for manufacturing and alignment. However, it exhibits considerable obstruction losses ( $\sim 16 \%$ including margins) inherent to catadioptric layouts, hindering the overall transmission. Along with cost and footprint, this will need to be factored in.

In the next months, while other trade-off analyses are carried out, the design team will further develop the overall optomechanical design towards its Delta-CoDR.

\section{ACKNOWLEDGMENTS}

This work was supported by the Programme National Cosmologie et Galaxies (PNCG) of CNRS/INSU with INP and IN2P3, co-funded by CEA and CNES. 


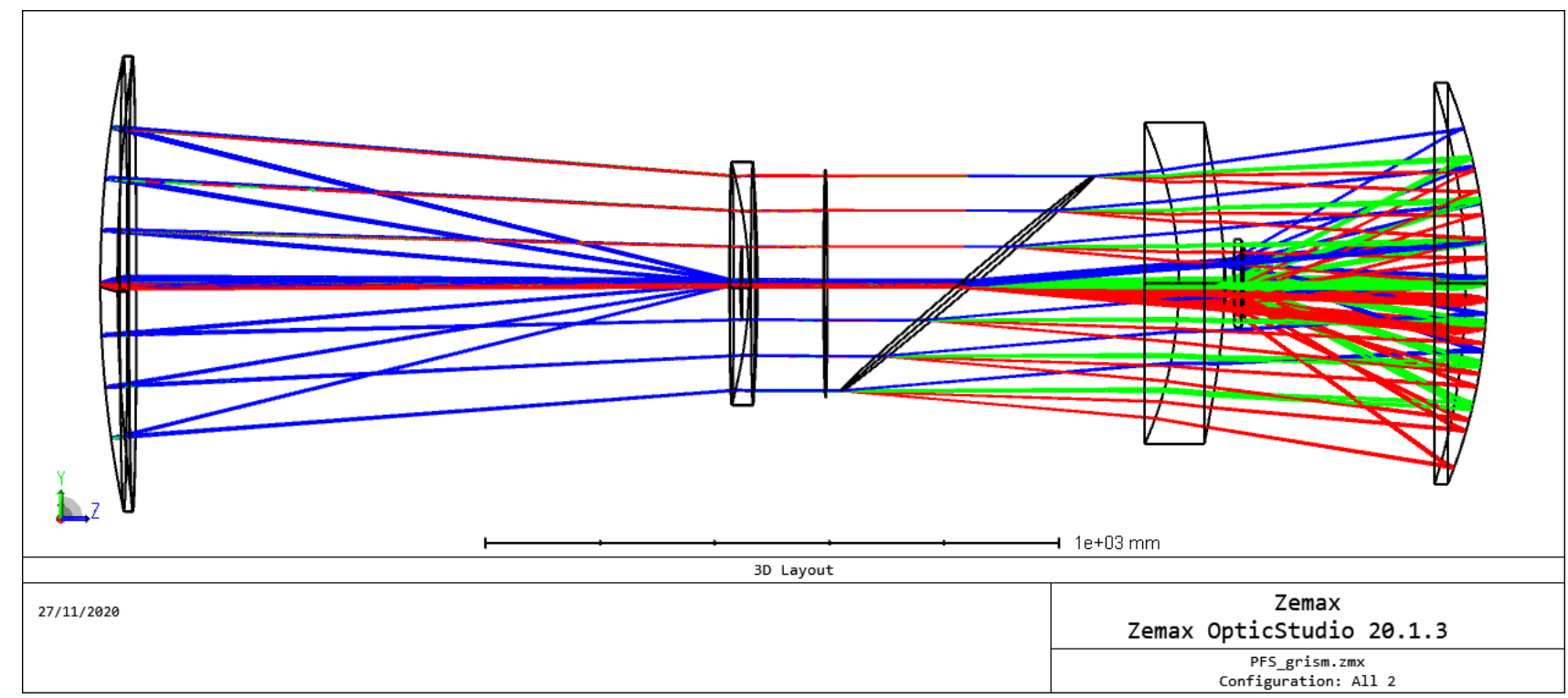

Figure 9. Optical layout of the cross-dispersed alternative design.

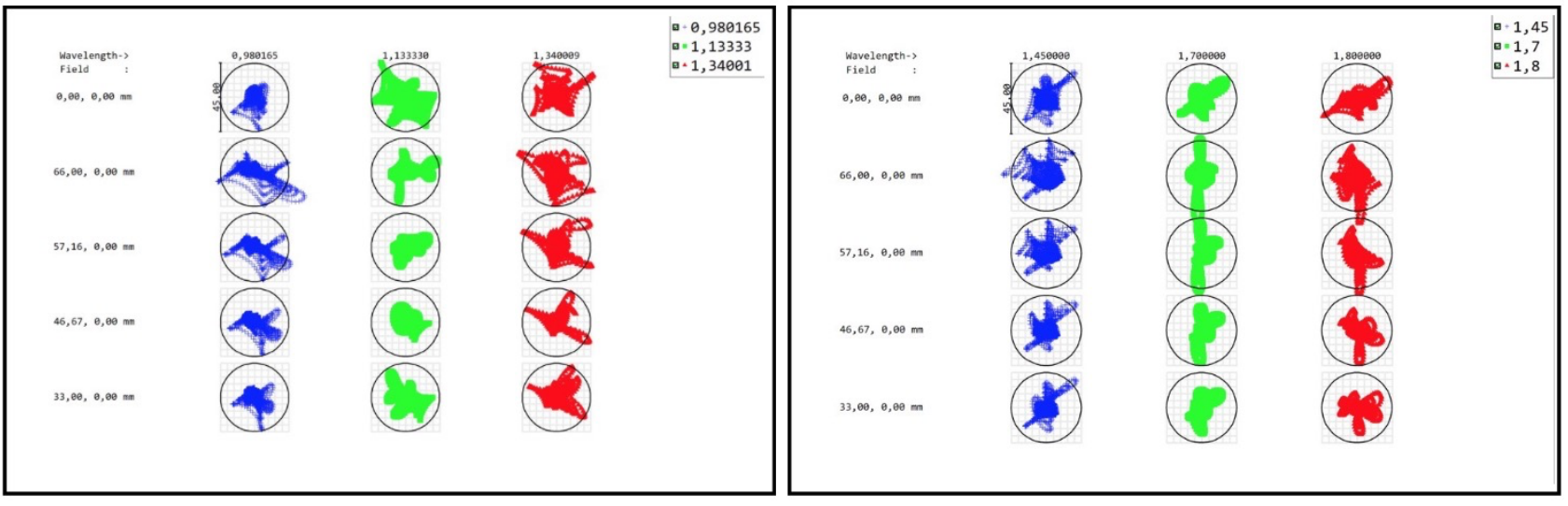

Figure 10. Spot diagrams of the cross dispersion preliminary concept.

\section{REFERENCES}

[1] Saunders, W., "Very fast transmissive spectrograph designs for highly multiplexed fiber spectroscopy," in Ground-based and Airborne Instrumentation for Astronomy VI, 9908, 2749 - 2760, SPIE (2016).

[2] Content, R. et al., "Optical design of the highly cost optimized new Hector Spectrograph," in Ground-based and Airborne Instrumentation for Astronomy VII, 10702, 2618 - 2631, SPIE (2018).

[3] Caillier, P. et al., "Maunakea spectroscopic explorer low moderate resolution spectrograph conceptual design," in Ground-based and Airborne Instrumentation for Astronomy VII, 10702, 2589 - 2608, SPIE (2018).

[4] Oliva, E. et al., "Toward the final optical design MOONS, the Multi-Object Optical and Near infrared Spectrometer for the VLT," in Ground-based and Airborne Instrumentation for Astronomy VI, 9908, 2277 - 2285, SPIE (2016).

[5] Delabre, B. and Oliva, E., "A full silica f/0.95 compact, achromatic and athermalized camera compatible with $60 \times 60 \mathrm{~mm}$ detector," Optical Design and Fabrication 2017 (Freeform, IODC, OFT) , ITh2A.5, Optical Society of America (2017).

[6] Barden, S. C. et al., "Astronomical applications of volume-phase holographic gratings," in 18th Congress of the International Commission for Optics, 3749, 52 - 53, SPIE (1999). 